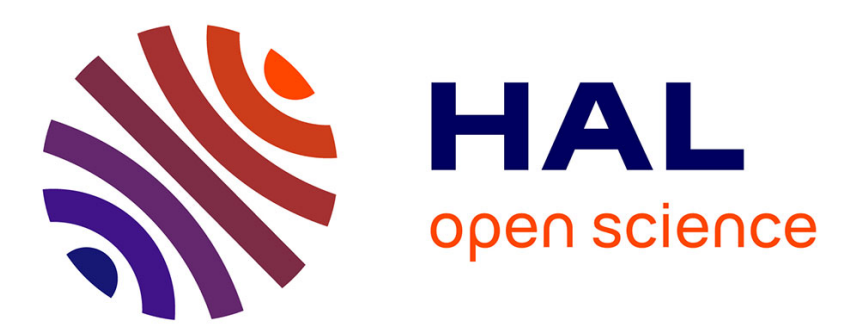

\title{
La réorganisation de l'action culturelle locale en Grèce: après la pratique de contractualisation, le moment des fusions de structures municipales
}

Dionysia Tzemopoulou

\section{- To cite this version:}

Dionysia Tzemopoulou. La réorganisation de l'action culturelle locale en Grèce: après la pratique de contractualisation, le moment des fusions de structures municipales. Pôle Sud - Revue de science politique de l'Europe méridionale, 2018, Varia, 2 (49), pp.71-89. halshs-01987349

\section{HAL Id: halshs-01987349 \\ https://shs.hal.science/halshs-01987349}

Submitted on 28 Jan 2019

HAL is a multi-disciplinary open access archive for the deposit and dissemination of scientific research documents, whether they are published or not. The documents may come from teaching and research institutions in France or abroad, or from public or private research centers.
L'archive ouverte pluridisciplinaire HAL, est destinée au dépôt et à la diffusion de documents scientifiques de niveau recherche, publiés ou non, émanant des établissements d'enseignement et de recherche français ou étrangers, des laboratoires publics ou privés. 


\title{
LA RÉORGANISATION DE L'ACTION CULTURELLE LOCALE EN GRÈCE : APRÈS LA PRATIQUE DE CONTRACTUALISATION, LE MOMENT DES FUSIONS DE STRUCTURES MUNICIPALES
}

\author{
Dionysia Tzemopoulou \\ Docteur en sciences politiques (Institut des Sciences Sociales du Politique/Université Paris \\ Nanterre) \\ Enseignante à l'Université Ouverte Hellénique (EAP) \\ d.tzemopoulou@yahoo.gr
}

\section{RÉSUMÉ/ABSTRACT}

La chute, en 1974, du régime de la dictature des colonels en Grèce a marqué le début d'une période de réformes du système politico-administratif. Les politiques étatiques de décentralisation culturelle ont contribué à l'évolution de l'action publique. Cet article propose une analyse du double processus de territorialisation et d'institutionnalisation des politiques culturelles. Dans un premier temps, il se focalise sur la structuration de l'action culturelle locale qui semble être associée à la gestion des formes renouvelées de coordination de l'action collective (Étatcommune) par le biais de la contractualisation. Dans un deuxième temps, la réorganisation de l'action publique territoriale repose, à partir de 2006, sur la fusion des structures culturelles municipales. A cet égard, nous abordons la redéfinition du rôle des protagonistes de l'action culturelle locale à travers l'analyse du parcours institutionnel des Entreprises municipales de développement culturel.

After the collapse of the military junta in 1974, a new political-administrative system was introduced in Greece. The period of reforms included new state policies of cultural decentralization, which advanced public action. This article attempts an analysis of the dual process of territorialization and institutionalization of cultural policies that was put forward during this period in Greece. First, the article discusses the ways that local cultural action was structured and associated, through contracting, with the management of renewed forms of coordination of collective action between the State and municipalities. Subsequently, starting in 2006, the reorganization of territorial public action was based on the merging of municipal cultural structures. In this respect, we propose a redefinition of the role of the key figures in local cultural action by focusing on the analysis of the institutional path of the Municipal enterprises for cultural development.

\section{MOTS-CLÉS/KEYWORDS}

Politiques culturelles, décentralisation, institutionnalisation, contractualisation, réorganisation, Grèce

Cultural Policies, decentralization, institutionalization, contracting, reorganization, Greece 
Les conditions politiques, sociales et économiques de la Grèce, dans l'environnement démocratique qui suivit la chute de la dictature des colonels (1974), furent à l'origine de profondes mutations dans la conception de l'intervention de l'État dans le domaine culturel. La perspective d'articuler la localité à l'espace public et de construire des politiques culturelles sectorielles entraîna une institutionnalisation de la politique culturelle au niveau local. L'offre publique de culture appuyée sur la nécessité de respecter les droits culturels et la citoyenneté stimula le processus de configuration de l'État-providence qui visait à la mise en œuvre d'un projet d'accès à la culture pour tous.

Dans le cadre de cet article, nous nous concentrons sur la culture contemporaine ${ }^{1}$, car elle nous offre la possibilité d'aborder les étapes de la décentralisation effectuées à partir des années 1980 jusqu'à nos jours. Autrement dit, les tentatives de décentralisation culturelle observées au cours de cette période reposèrent sur l'établissement de politiques contractuelles dont la culture contemporaine fut l'objet essentiel. Les relations des partenaires, État-communes, furent refondues par la nécessité d'évolutions institutionnelles imposées tant par les politiques de décentralisation que par l'intégration du pays à la CEE en 1981. En outre, pendant cette période, la question de la clarification des obligations et des rôles des différentes collectivités publiques dans le domaine culturel fut posée pour la première fois de manière systématique.

Le défi représenté par le passage de la décentralisation de l'action culturelle à sa territorialisation et, précisément, à sa municipalisation, imposa une philosophie de coopération régissant les pratiques contractuelles. Au cours des années 1980 et 1990, l'État sollicita des communes désireuses de participer au pilotage et au financement des activités culturelles au niveau régional tandis qu'un grand nombre de municipalités revendiquèrent le label d'État, en participant soit à l'institution des Théâtres Municipaux et Régionaux (DIPETHE) (voir annexes tableau $\mathrm{n}^{\circ}$ 2) soit au Réseau Culturel National des Villes (EPDP) (voir annexes tableau $\mathrm{n}^{\circ} 3$ ). Cependant, l'édification des politiques culturelles municipales fut, dans une large mesure, fondée sur la création des Entreprises municipales de développement culturel.

La mission de ces structures consistait à distinguer la double nature de la décentralisation : artistique et administrative. Une décentralisation artistique, traduite par la diffusion de la culture en province, c'est-à-dire en dehors d'Athènes et de Thessalonique ; et une décentralisation administrative fondée sur l'effort de transfert de compétences relevant de l'État aux collectivités

${ }^{1}$ Ce domaine d'action culturelle regroupe les arts du théâtre, de la danse, de la musique, du cinéma ainsi que les arts plastiques et le livre. 
territoriales. Ainsi, «la décentralisation a confirmé le rôle des communes comme les premières entrepreneurs du pays » puisque la politique culturelle devint, à partir des années 1980, «un domaine d'action publique partagée entre l'État et les villes » pour reprendre les termes de Guy Saez (Saez, 2001, p. 156).

Les politiques et pratiques de la décentralisation prirent dans le domaine culturel différentes orientations selon les époques. La coopération contractuelle fut conçue en tant que forme de décentralisation. Elle incita des communes à prendre en charge les institutions culturelles et à les développer. Néanmoins, tant les communes que l'État cherchaient depuis la moitié des années 2000 à redéfinir leurs rôles. Le Code des Communes et Communautés en 2006 (Loi $\mathrm{n}^{\circ}$ 3463/2006) et le Programme Kallikratis en 2010 (Loi n 3852/2010) conduisirent à un nouveau type d'expérimentation. Dans ce contexte, les questions qui guident la réflexion proposée dans cet article sont les suivantes : quelles sont les priorités des politiques culturelles locales à partir de 2006? Quelles sont les transformations des rapports entre les différents échelons (État, communes)? Et, en dernier lieu, quelles sont les nouvelles modalités d'instrumentation de l'action publique culturelle? L'analyse du parcours institutionnel des Entreprises municipales de développement culturel sera l'angle sous lequel nous allons les aborder. Dans cette optique, nous souhaitons comprendre dans quelle mesure ce nouveau cadre législatif se fonde sur un processus simple de transition d'un stade à l'autre des politiques culturelles ou bien sur un processus de valorisation de l'action publique.

\section{LE LANCEMENT DES ENTREPRISES MUNICIPALES DE DÉVELOPPEMENT CULTUREL : L'APPROCHE HISTORIQUE}

\section{Le mécanisme polyvalent de la décentralisation culturelle}

En 1981, l'alternance politique en Grèce a porté, pour la première fois dans la période d'aprèsguerre, un parti socialiste (PASOK) au gouvernement. Cette nouvelle époque fut marquée par la revendication de démocratisation entendue comme synonyme d'État-providence. Cette demande politico-sociale, exprimée par la base électorale du PASOK, détermina le contenu des réformes entreprises par les nouveaux élus dans les années quatre-vingt. Les actions en faveur des groupes défavorisés, visant à l'abolition des injustices, avaient pour ambition d'établir une nouvelle conception des rapports entre les citoyens et l'État. Depuis les années soixante-dix, le PASOK 
avait déjà accordé une attention particulière à l'administration locale en la considérant, avec le syndicalisme et le parlement, comme l'une de trois composantes de la démocratie.

C'est ainsi que dans le courant des années quatre-vingt fut amorcée la création d'entreprises appartenant aux collectivités locales dans le but de contribuer au développement social et économique de la périphérie. Nicolas-Komnemos Hlepas constate que la décentralisation territoriale « avança grâce au prolongement progressif de ses compétences, à l'adaptation de nouvelles formes d'organisation " (Hlepas, 2003, p. 68), comme celle des entreprises municipales. De plus, il qualifie les entreprises de modèle de "privatisation organisationnelle » attaché à un type de management public, tout en remarquant qu'elles s'étaient aussi révélées un moyen efficace de développement local, quand ce dernier était lié aux succès d'initiatives de maires, comme le fut celle de Stavros Bènos, à Kalamata (Hlepas, 2010, p. 585). Leur mission s'appuya notamment sur le secteur tertiaire, comme dans le reste de l'Europe (Suède, Italie, France, Angleterre) où cette stratégie avait été lancée à partir des années soixante (Iliopoulou, 1989). L'accroissement impressionnant de leur nombre - dû à leur caractère public susceptible de garantir une répartition équitable des résultats économiques des activités d'investissement des collectivités locales - eut tendance à atténuer les disparités régionales et sociales.

De ce fait, le début des années quatre-vingt vit un nombre limité d'entreprises se concentrer presque exclusivement sur l'eau et l'assainissement ${ }^{2}$ tandis que leur cadre institutionnel (concernant leurs établissement et financement) devint plus spécifique à partir de $1984^{3}$. En d'autres termes, le Code municipal et communautaire de 1985 obligeait les communes à la constitution d'entreprises visant autant à la mise en place de projets au profit du public qu'à la production de biens et services, alors qu'il ne manquait pas de faire référence à la nécessité de réaliser des bénéfices ${ }^{4}$. Nous constatons donc que les gains ne constituèrent pas le seul but des entreprises qui apparurent davantage comme une unité de production de biens et de services sociaux. Ici, il convient de mentionner que le domaine de la culture fit partie des actions essentielles des communes au cours de cette période. La constitution et le fonctionnement des centres culturels, des bibliothèques, des musées et des activités de loisirs s'inscrivaient au rang de leurs priorités. L'évolution de ces établissements comme personnes juridiques autonomes et, en

\footnotetext{
${ }^{2}$ Voir Loi n ${ }^{\circ}$ 1069/1980 (articles 205-225).

${ }^{3}$ Plus précisément, le cadre institutionnel pour le fonctionnement des entreprises municipales fut établi par la Loi $\mathrm{n}^{\circ}$ 1416/1984 (articles 35-39, 42, 46) et le décret présidentiel $n^{\circ} 76 / 1985$, alors que la loi de 1622/1986 décrivait le cadre institutionnel pour le fonctionnement des collectivités locales, leurs échelons, leurs responsabilités et leur rôle dans le développement régional et la planification démocratique.

${ }^{4}$ Source : Code municipal et communautaire de 1985, Arrêté présidentiel n 76/1985.
} 
particulier, l'adoption du statut d'entreprise municipale ${ }^{5}$, donna aux municipalités beaucoup de souplesse dans l'administration, la gestion et la sélection du personnel et des ressources financières.

Dans ces conditions, la majorité des communes décidèrent de créer, à partir de 1985, des Entreprises municipales de développement culturel, en profitant des dispositions favorables de la loi $\mathrm{n}^{\circ} 1262 / 1982^{6}$. Ces structures municipales polyvalentes proposaient un projet de développement culturel destiné à la population locale. Plus concrètement, des ateliers d'arts (théâtre, danse, arts plastiques), des conservatoires, l'organisation de manifestations culturelles variées (p. ex., promotion du patrimoine populaire, activité éditrice), des infrastructures culturelles, régissaient la logique de leur fonctionnement (voir annexes, encadré $\mathrm{n}^{\circ} 4$ ). La dynamique que ces structures acquirent progressivement dans les années suivantes les plaça au centre du processus de planification d'une politique culturelle au niveau local. L'instrument administratif des contrats de programme devint l'outil indispensable pour l'évolution des relations contractuelles entre les communes et l'État, et pour l'institution même des Entreprises municipales de développement culturel. Le premier contrat de ce type fut signé par l'État, en 1985 avec la municipalité de Kalamata ${ }^{7}$. Il constitua la base rédactionnelle de la totalité des textes similaires dans tout le pays, tandis qu'il proposait de nouvelles formes de coopération entre gouvernement central et local.

\footnotetext{
${ }^{5}$ Le statut des entreprises municipales fut également adopté par les Théâtres Municipaux Régionaux.

${ }^{6}$ Extrêmement importante pour le renforcement de cette nouvelle institution fut la contribution de la loi $\mathrm{n}^{\circ}$ 1262/1982, qui prévoyait des dispositions favorables pour le financement des entreprises municipales. En effet, selon la région et le type d'entreprise, des entreprises municipales pouvaient être subventionnées à hauteur de $65 \%$ du coût total de l'investissement.

${ }^{7}$ Selon le premier contrat de programme (1985), l'Entreprise municipale de développement culturel de Kalamata (DEPAK) était dirigée par un Conseil de onze membres dans la perspective d'une gestion pluraliste et démocratique fondée sur la représentation de plusieurs agents locaux de nature politique, administrative et artistique. Ainsi, son Conseil d'administration était composé (en plus des trois membres du conseil municipal), d'un représentant du personnel de l'entreprise, de représentants (cinq membres) des organismes culturels et sociaux de la ville tandis que deux membres du conseil de l'entreprise provenaient des résidents de la municipalité de Kalamata ayant une expérience et des connaissances relatives à l'objet de la DEPAK. Dans le cadre du second contrat de programme (1996), le Conseil d'administration de l'Entreprise municipale fut réduit à sept membres (ajouté d'un représentant du ministère de la Culture). La nouvelle constitution était la suivante : deux conseillers municipaux, un représentant du ministère de la Culture (par décision du ministre de la Culture), deux citoyens de la commune ayant une expérience et des connaissances relatives à l'objet de l'entreprise, un représentant des salariés de l'entreprise, un représentant d'une institution culturelle de la ville. Les ressources pour le fonctionnement de l'Entreprise municipale étaient de diverses provenances, selon le contrat de programme de 1985. Au-delà de la subvention attribuée par les parties contractantes (ministère de la Culture, secrétaire d'État à la Jeunesse, municipalité de Kalamata), ses ressources étaient complétées par des recettes d'écoles municipales (Conservatoire, Atelier d'arts plastiques, École de danse) et les tickets d'entrée aux manifestations culturelles organisées par la DEPAK. Le financement par d'autres acteurs du secteur public, par le programme d'investissements publics ou par des fonds européens pouvait compléter le revenu de l'entreprise. Source : archives du ministère de la Culture, Direction Générale de la Culture Contemporaine, Département du Théâtre et de la Danse, Dossier thématique - Contrats de programme des DIPETHE.
} 
Cependant, la multiplication des entreprises municipales, qui devait favoriser l'autonomie des communes - en les aidant à affirmer leur ambition de souveraineté politico-administrative n'alla pas dans le sens d'une gestion rationnelle. Dans ce contexte, de nouvelles composantes de l'action publique émergèrent. Elles permirent de mieux comprendre les raisons qui induisirent un changement du cadre de fonctionnement des entreprises municipales, à partir du milieu des années 2000. Ces modifications furent intégrées dans la réforme du Code des Communes et Communautés en 2006 (Loi n 3463/2006), et furent la source de mutations considérables dans l'exercice de la politique culturelle régionale, qu'illustre encore le bouleversement radical de l'image politico-économique du pays.

\section{LA MODIFICATION DU CADRE INSTITUTIONNEL RÉGISSANT L'ACTION PUBLIQUE TERRITORIALE : LA FUSION DES STRUCTURES CULTURELLES MUNICIPALES}

\section{Le Code des communes et communautés (2006) et le Programme Kallikratis (2010) : le bouleversement du cadre législatif en faveur des réformes politico- administratives}

Dans les années 2000, les modèles de légitimation de l'intervention publique, utilisés principalement par l'État et dans une moindre mesure par les collectivités locales, subirent des modifications par rapport aux décennies précédentes. Les orientations des politiques culturelles nationales ne privilégièrent plus l'action culturelle locale. Le changement radical du paysage culturel, à partir de 2006, se traduisit, d'une part, par le renforcement des compétences des communes et des régions en matière culturelle, et, d'autre part, par une politique généralisée de fusions, le Code des communes et communautés (2006) ainsi que le Programme Kallikratis (2010) gouvernant ces transformations. L'évolution de l'institutionnalisation des politiques culturelles locales semble découler de la marginalisation des instruments traditionnels de l'action publique tels que les contrats de programme, et d'un effort pour rétablir les collectivités locales dans leurs prérogatives d'action culturelle européenne, mais sans le soutien de l'État. Il y avait là matière à bouleverser la nature des relations entre les communes et l'État qui jusque-là reposaient sur un partenariat contractuel.

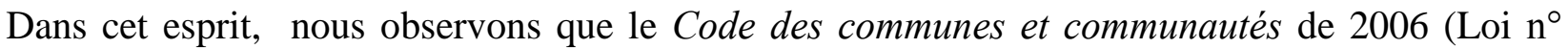
3463/2006) et le Programme Kallikratis de 2010 (Loi $\mathrm{n}^{\circ}$ 3852/2010) ont renouvelé les compétences des collectivités territoriales en matière culturelle. Les nouvelles dispositions 
légales ont, dans une certaine mesure, réformé leurs obligations et leurs rôles dans la perspective d'une meilleure coordination de l'action publique. Les deux lois résultèrent des pressions européennes qui encourageaient les programmes de réforme au niveau politico-administratif. Il convient ici de mentionner que le défi résidait dans l'application des réformes et dans la conciliation des pressions de l'UE et des besoins internes du pays (Featherstone, 2007). Dans cette optique, leur efficacité dépendit de l'attitude de leurs supporters à l'intérieur du pays.

C'est ainsi que la redéfinition des compétences des collectivités locales fut également susceptible d'exprimer un effort pour aplanir leurs conflits avec l'État en évitant les chevauchements et les lacunes politiques caractéristiques des décennies précédentes. Cette initiative eut un impact direct sur la gestion des affaires culturelles au niveau régional, et, en particulier, sur le fonctionnement des Entreprises municipales de développement culturel. En outre, l'enrichissement des compétences en matière culturelle des communes et des régions visant au renforcement de leur rôle décisionnel posa la question de la capacité à gouverner (capacity to govern) (Passas, 2009). Guy Peters a attribué à ce sens la capacité de coordination de l'action publique considérée comme agent nécessaire pour garantir la cohérence de cette dernière (Peters, 1998). De même, il n'a pas manqué de souligner le lien entre cette capacité et le processus de sélection des mérites des objectifs de la politique à suivre.

\section{Encadré 1. Les dispositifs du Code des communes et communautés de 2006 sur la culture}

Les responsabilités des communes et communautés concernent :

- La mise en œuvre des politiques de protection de la culture locale.

- La promotion de l'action culturelle et artistique produite localement à travers la création des centres culturels, des musées, des galeries, des cinémas, des théâtres, des orchestres philharmoniques, des écoles de musique, de danse, de peinture, de sculpture etc.

- L'organisation de concerts, de représentations théâtrales et autres manifestations culturelles organisées par euxmêmes ou auxquelles ils participent.

- La création et le fonctionnement des bibliothèques.

- La protection des musées, des monuments, des grottes et des sites archéologiques et historiques.

- Le développement du tourisme culturel.

- La promotion des échanges culturels au niveau national, européen et international.

Source : Loi n 3463/2006 (article 75, paragraphe I). 


\section{Encadré 2. Les dispositifs du Programme Kallikratis (2010) sur la culture}

L'article $\mathrm{n}^{\circ} 75$ (paragraphe I) de la loi $\mathrm{n}^{\circ}$ 3463/2006 a été complété, en 2010, par les dispositifs suivants du Programme Kallikratis destinés à élargir les compétences des communes :

-L'organisation de concerts et autres manifestations culturelles pour les étudiants, après approbation de la commission scolaire.

-L'octroi de permis pour l'établissement et le fonctionnement des institutions privées musicales (conservatoires, écoles de musique, chorales, orchestres symphoniques et ensembles de musique de chambre).

-La participation d'un représentant de la municipalité dans le comité organisateur des festivals locaux.

-Le financement des agents qui développent des activités culturelles dans la compétence territoriale de la municipalité et de ceux qui contribuent au développement et à la promotion du tourisme, à la suite d'une décision du conseil municipal à la majorité des deux tiers $(2 / 3)$ de ses membres.

Source : Loi $\mathrm{n}^{\circ}$ 3852/2010 (article 94).

\section{La politique des fusions : une réorganisation de l'action publique territoriale}

Après la réforme de Kapodistrias en 1997 (Loi n ${ }^{\circ}$ 2539/1997) ${ }^{8}$, le Programme Kallikratis initia une seconde vague de fusions des communes et communautés afin de créer des communes fortes dans les régions grecques, tandis que cette loi englobait également les régions métropolitaines d'Athènes et de Thessalonique. Il convient de noter que, jusqu'à 2010, 53\% des communes de Grèce comptaient des populations de moins de 5.000 habitants alors que 25\% des municipalités en comptaient $10.000^{9}$. Dans ce contexte, l'État tenta une réorganisation territoriale, administrative et principalement fonctionnelle du pays dans le but de renforcer sa planification démocratique et son potentiel de développement. L'objectif essentiel de cette amélioration fonctionnelle se traduisit par l'effort de l'État grec d'économiser les ressources par la réduction du nombre des collectivités locales $\left(\mathrm{OTA}^{10}\right)$ et de leurs entités, ce qui devait permettre une gestion simplifiée.

\footnotetext{
${ }^{8}$ Les propositions du «programme I. Capodistrias» (Loi $n^{\circ}$ 2539/1997) basées sur la fusion des communes et communautés ont modifié l'organisation territoriale de la Grèce entre 1997 et 2010 quand le Programme Kallikratis (Loi $n^{\circ} 3852 / 2010$ ) bouleversa à nouveau la carte territoriale du pays. Dans le cadre de la réforme proposée par la loi $\mathrm{n}^{\circ}$ 2539/1997, 900 communes et 133 communautés ont été créées, alors que, initialement, le nombre de communes s'élevait à 5389, et celui des communautés à 377. Néanmoins ce programme concernait la fusion des petites communes et communautés qui ne disposaient pas de structures culturelles en tant qu'Entreprises municipales de développement culturel, les DIPETHE et les structures d'EPDP. Dans cet esprit, nous n'avons pas entrepris une analyse plus détaillée de cette réforme. Pour la présentation du Programme I. Capodistrias voir (Hlepas, 2008) ; (Chatzipantelis, 2008); (Psycharis \& Simatou, 2003).

${ }^{9}$ Ministère de l'Intérieur, de la Décentralisation et du Gouvernement Électronique, Rapport d'évaluation du projet de loi sur le Programme Kallikratis, Athènes, 2010.

${ }^{10}$ Organismes d'Administration Locale.
} 
Dans cette perspective, nous observons que les services publics des communes appelées à former une nouvelle municipalité élargie fusionnèrent obligatoirement en un seul service public ${ }^{11}$. Rappelons ici le rôle social des Entreprises municipales de développement culturel par l'entremise du rapport d'évaluation du projet de loi sur le Programme Kallikratis élaboré en 2010 par le ministère de l'Intérieur, de la Décentralisation et du Gouvernement Électronique : «Ces nouvelles municipalités pourraient répondre à un nouveau grand défi pour l'administration locale du pays lié à leur rôle important et actif dans la fourniture de services sociaux ${ }^{12}$. Le rapport soulignait également que les pays européens disposant d'un État-providence fort avaient une administration locale efficace qui comptait parmi ses priorités la fourniture de services sociaux.

Ces fusions furent considérées comme nécessaires pour diverses raisons. Un rapport d'évaluation réalisé cette fois par l'Institut de l'administration locale (ITA) en 2006 sur la rationalisation des OTA, indiqua les principales causes du caractère pathogène des entreprises d'OTA parmi lesquelles les Entreprises municipales de développement culturel. L'ITA présentait ses conclusions pour la période du début de leur constitution (dans les années 1980) jusqu'en 2005 , c'est-à-dire avant l'adoption des dispositions du Code des communes et communautés de 2006 différenciant les conditions de leur fonctionnement. Le rapport indiquait que tant leur cadre législatif lâche - qui créait pour eux un champ presque illimité d'action (ou d'expérimentation) - que le faible degré de leur supervision par des mécanismes de contrôle de l'État avaient conduit dans de nombreux cas à l'inefficacité de leur action ${ }^{13}$. Ainsi, parmi les conséquences qu'il mettait en évidence figuraient, dans un premier temps, l'existence d'un trop grand nombre d'entreprises dans toute la Grèce, et dont le bilan économique, pour beaucoup d'entre elles, n'était pas positif. Dans un deuxième temps, il permettait de constater l'implication des OTA dans les affaires alors qu'elles étaient dénuées d'expertise et de savoir-faire. Enfin, selon les conclusions d'ITA, les entreprises d'OTA avaient sous-estimé l'efficacité des procédures d'évaluations destinées au recrutement du personnel.

\footnotetext{
${ }^{11}$ Par décision du conseil municipal sont définis le nom, le but, la gestion, le capital, les ressources, la durée et le siège du service (et toute autre information nécessaire à la discrétion du conseil municipal). Les biens meubles et immeubles des services fusionnés sont automatiquement transférés au nouveau service en tant que successeur de tous les droits et obligations des services fusionnés y compris les contrats de travail à durée déterminée jusqu'à l'échéance. Voir Loi n 3852/2010 (article 109).

${ }^{12}$ Ministère de l'Intérieur, de la Décentralisation et du Gouvernement Électronique, Rapport d'évaluation du projet de loi sur le Programme Kallikratis, op. cit., p.106.

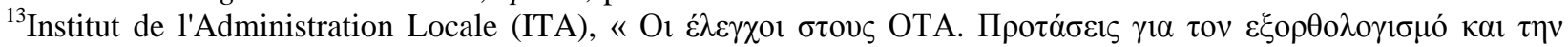
$\alpha \pi \circ \tau \varepsilon \lambda \varepsilon \sigma \mu \alpha \tau$ système], Athènes, le 22.06.2006.
} 
Quant à ce dernier point, il convient de mentionner que selon les données du Registre des employés des OTA en 2002, 20\% seulement parmi eux étaient diplômés de l'enseignement supérieur ou technologique (Tsioumas, 2011). La majorité de leurs employés avaient achevé l'école primaire ou le lycée mais n'étaient pas allés au-delà. Cette situation fut bouleversée par la loi $\mathrm{n}^{\circ} 3812$ de 2009 concernant la réforme du système de recrutement du secteur public. De ce fait, la responsabilité du recrutement de personnel permanent et saisonnier des OTA fut assumée par le Conseil suprême pour la sélection du personnel civil (ASEP) ${ }^{14}$. Le recrutement des artistes constitue toutefois un cas exceptionnel (contrats de projets). Il est à noter que ce nouveau cadre législatif orienté également vers le renouvellement du personnel des municipalités par des fonctionnaires sélectionnés en fonction de leurs mérites (personnes qualifiées) fut considéré à la fin des années 2000 comme une mesure contribuant à l'amélioration des conditions de la planification et de la mise en place des politiques culturelles locales.

\section{L'impact des fusions sur les Entreprises municipales de développement culturel}

Avant l'adoption du programme Kallikratis, les petites municipalités n'étaient pas en mesure de subvenir aux besoins de leurs propres Entreprises de développement culturel. Ainsi, la loi $\mathrm{n}^{\circ}$ 3852 de 2010, concrétisant ce programme, initia la création de municipalités élargies et contribua au fonctionnement efficace de ces structures des OTA. Cependant, le dispositif de la loi (article 103) qui donnait à chaque commune le droit de former jusqu'à deux entités publiques provoqua en 2010 le mécontentement des agents locaux. Avant d'analyser ces réactions nous devons mentionner que la loi précisait les domaines d'action ${ }^{15}$ des deux entités publiques : l'une pouvait concerner la protection sociale et l'éducation, et l'autre la culture, le sport et l'environnement ${ }^{16}$. Si la municipalité disposait déjà d'un service public, elle n'avait droit qu'à la création d'une seule entité publique. Par exception, les communes de plus de 300.000 habitants avaient le droit de créer des entités publiques supplémentaires. Cette disposition de la loi ne satisfit ni les fonctionnaires des structures fusionnées ni les professionnels de la culture. Plus précisément, le fait que la loi ne donnait pas la possibilité d'accroître le nombre des entités publiques indépendamment de la population des communes, fut considéré comme une entrave au développement culturel local (Kabouris, 2014). L'argument principal de cette position reposait

\footnotetext{
${ }^{14}$ Loi n ${ }^{\circ} 3812 / 2009$.

${ }^{15}$ Le contenu exact de ces domaines était prévu par la Loi n ${ }^{\circ} 3463 / 2006$ (article 75).

${ }^{16}$ Loi n ${ }^{\circ} 3852 / 2010$ (article 102).
} 
sur le constat que les objets de l'action publique - culture, protection sociale, athlétisme étaient de nature hétérogène, ce qui entravait leur coordination au sein d'une seule structure. Autrement dit, leur disjonction en différentes structures apparaissait nécessaire pour améliorer les services fournis.

Dans ce contexte, la création d'unités distinctes au sein des communes, soit comme entreprise de développement culturel soit comme service public (depuis 2006), constitua une demande constante de la part des agents locaux dans le courant de la période 1980-2010. L'étendue des compétences des communes impliquait d'ailleurs une telle politique de gestion de leurs affaires. Néanmoins le défaut de reconnaissance, de la part de l'État, du double problème de l'organisation de l'action culturelle au niveau local, c'est-à-dire de la concentration excessive des services au sein d'une structure d'une part, et l'augmentation illimitée du nombre de ces derniers d'autre part, furent les causes fondamentales des problèmes posés par les politiques de réforme en Grèce. Calliope Spanou, dans son analyse des raisons de cette faiblesse soulignait «L'impuissance à surmonter les problèmes déjà connus depuis des décennies, se réfère à ce qui peut être qualifié de dépendance au chemin emprunté. La nouvelle a tendance à être absorbée et/ou déterminée par l'ancien » (Spanou, 2001, p. 313).

Elle ajoutait que les réformes étaient parfois liées à un processus de légitimation des options politiques qui incorporait les déficits politiques précédents. Processus étayé tant par les objectifs imprécis des réformes que par le manque de préparation adéquate pour leur mise en œuvre. De ce fait, bien que les politiques de fusions fussent présentées par l'État comme prometteuses sur le plan de l'initiation politique et de la formulation des politiques (policy initiation, policy formulation) (Melissas, 1995), elles n’apportèrent pas les résultats escomptés. La réduction du nombre des Entreprises municipales de développement culturel par le biais de leur fusion fonctionna en tant que mécanisme de contrôle adopté par l'État afin de rationaliser leur action. Cependant, tout à la fois la méfiance avec laquelle ces mécanismes furent perçus par les agents locaux, et la mauvaise évaluation de l'importance des conditions de fonctionnement des nouvelles structures (services publics) de compétences étendues, annulèrent les effets de cet effort de réforme.

\section{L'affaiblissement du partenariat contractuel : quel avenir pour la coopération État-collectivités locales?}

Les contrats de programme, instrument essentiel pour l'exercice de la politique culturelle régionale en Grèce à partir des années 1980, furent progressivement marginalisés dans les années 
2000. Dans un premier temps, l'abolition de l'institution du Réseau Culturel National des Villes (EPDP) en 2002 entraîna, d'une part, la rupture du réseau et, d'autre part, le fonctionnement indépendant d'une minorité seulement de ses structures qui poursuivirent leur action au cours de cette décennie. Dans ce contexte, il est à noter qu'à partir du milieu des années 2000, le ministère de la Culture ne participa pas, dans le cadre d'une relation contractuelle, à leur financement. Dans un deuxième temps, ce type de coopération, entre les collectivités locales et l'État, fut également abandonné dans le cas des Entreprises municipales de développement culturel. Ce partenariat avait atteint sa limite, avant même la mise en place de la politique de leur fusion. La stagnation progressive du budget étatique conjointement au changement des priorités des politiques culturelles détourna l'attention de l'État vers le centre. Athènes et Thessalonique monopolisèrent son intérêt.

En revanche, les structures du réseau des Théâtres Municipaux Régionaux (DIPETHE) se seront réservés le privilège de la coopération contractuelle avec le ministère de la Culture sans pour autant qu'aient prévalu les mêmes conditions. La réduction progressive de la durée des contrats de programme des DIPETHE depuis les années 1980 témoigna aussi bien de la difficulté de l'État à respecter ses obligations en tant que partie contractuelle que de sa volonté d'éviter tout engagement à long terme. Ainsi, nous observons que la durée des contrats de programme signés en 2009 fut limitée à dix-huit mois tandis que les années suivantes (2011 et 2012) la virent fondre jusqu'à six mois ${ }^{17}$.

Tableau 1. La durée des contrats de programme des Théâtres Municipaux Régionaux (1985-2010)

\begin{tabular}{|c|c|}
\hline Période de validité & Durée (nombre d'années) \\
\hline $1980-1990$ & 10 \\
\hline $1990-2000$ & 6 \\
\hline $2000-2010$ & 5 \\
\hline $2010-2020$ & 6 mois -1 an \\
\hline
\end{tabular}

Source : archives du ministère de la Culture, Direction générale de la culture contemporaine, Département du théâtre et de la danse, Dossier thématique - Contrats de programme des DIPETHE [traitement des données par l'auteure de l'article].

Dans ce contexte, nous aurions pu nous attendre à ce que l'État veuille se désengager complètement du partenariat contractuel. Néanmoins, en 2010, le Programme Kallikratis concevait ainsi l'implication du ministère de la Culture : «Dans le cas où les projets ou les

\footnotetext{
${ }^{17}$ Source : archives du ministère de la Culture, Direction Générale de la Culture Contemporaine, Département du Théâtre et de la Danse, Dossier thématique - Contrats de programme des DIPETHE.
} 
services prévus dans le cadre des contrats de programme sont de nature culturelle: la promotion, la protection et la conservation des monuments, l'intervention dans les domaines des bâtiments ou implantations traditionnelles, la préservation et la diffusion des éléments de la culture, la construction des espaces culturels, le soutien des activités et des manifestations liées aux sciences, aux lettres et aux arts, le ministère de la Culture participe en tant que partie contractuelle $»^{18}$.

Ce dispositif montrait l'intention de l'État de continuer à contrôler ou surveiller la vie culturelle $\mathrm{du}$ pays. Bien que le nombre des contrats de programme de développement culturel a sérieusement diminué à la fin des années 2000, le ministère de la Culture continua de s'impliquer dans le déroulement de l'action publique au niveau régional. Nous observons toutefois que la nature de la pratique de partenariat contractuel avait changé puisque les contrats de programme ne pouvaient plus garantir le financement croisé qui aurait dû être assumé par tous les partenaires concernés, du fait de l'instabilité financière du pays, évidente depuis la fin de la décennie 2000. En deuxième lieu, l'attribution de nouvelles compétences culturelles aux régions et aux communes à travers le Programme Kallikratis soulignait la contradiction encore plus aiguë de cette attitude du ministère de la Culture. D'une part, nous constatons que la confiance de l'État envers les autres échelons politico-administratifs se traduisit par les dispositifs de la loi n 3852 de 2010 tels que nous les avons analysés ci-dessus, et d'autre part, qu'il peinait à identifier son nouveau rôle.

Dans ces conditions, nous n'examinons pas seulement la dévalorisation des contrats de programme de développement culturel en tant qu'instruments de l'action publique mais nous recherchons également ce que doit représenter le partenariat. Ici, il convient de mentionner que René Rizzardo, en 2005, dans son approche du paysage des responsabilités culturelles en France, écrivait : "Le partenariat, souvent contractuel, a retardé des mesures de décentralisation plus radicales » (Rizzardo, 2005, p. 29). Nous ne pouvons pas dire qu'en Grèce ce soit en faveur d'un effort d'aménagement et de coordination du territoire que la pratique des contrats de programme fût marginalisée. Bien que le ministère de la Culture ait ambitionné en 2012 de mettre en place un projet institutionnel visant «à sa coopération avec les régions et les municipalités du pays, à travers une structure flexible qui prendrait en charge à la fois la planification stratégique du pays, effectuée au niveau central et les objectifs spécifiques de chaque Région et de chaque

\footnotetext{
${ }^{18}$ Loi n $^{\circ} 3852 / 2010$ (article 100).
} 
municipalité $\gg{ }^{19}$, les conditions politico-économiques du pays dans le courant de cette période accrurent la difficulté d'une telle planification.

\section{Encadré 3. Les points principaux pour un renouvellement de la politique culturelle régionale en 2012}

« La proposition du ministère de la Culture pour une nouvelle politique culturelle, telle que définie dans un document pertinent de soixante - dix pages en 2012, planifia la création d'un Conseil de culture contemporaine dans chaque Région et commune, qui devait fonctionner en conformité avec les normes initiées par le département de la Politique culturelle régionale du ministère. En outre, elle suggéra l'établissement d'un Bureau de Planification Culturelle et de Programmes. Ce Bureau devait doter tout d'abord le personnel de la Région ou de la municipalité où son siège était situé, tandis que son directeur voyait ses compétences augmentées, précisées dans l'avis pertinent de la Région ou de la municipalité suite aux suggestions faites par le ministère de la Culture (Département de la Politique culturelle régionale). Les deux nouvelles institutions/structures devaient définir un Plan opérationnel culturel régional ou municipal de trois ans, à approuver par le Conseil régional ou le Conseil municipal. Ces Plans devaient être évalués par le Département de la Politique culturelle régionale du ministère de la Culture afin de vérifier si les critères de convergence - reposant sur les orientations générales du ministère quant à l'exercice de sa politique tant au niveau régional que central - étaient respectés ».

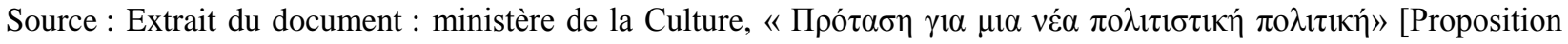
pour une nouvelle politique culturelle], Athènes, mars 2012, p. 58-64 [traduction par l'auteure de l'article].

Au-delà de ce constat, c'est-à-dire celui du bouleversement des conditions dans lesquelles s'effectua le partenariat entre l'État et les collectivités territoriales dès la fin des années 2000, nous devons considérer la logique qui régit cette réforme. Le Programme Kallikratis avait mis l'accent sur la clarification des responsabilités culturelles des régions, des communes et de l'État. Néanmoins, il convient de mentionner ici que le partenariat contractuel ne correspondait plus à l'effort de «multiplication des niveaux de responsabilité administrative et politique 》 pour reprendre une formule de Jean-Pierre Gaudin (Gaudin, 2004, p. 216). Les collectivités locales ne revendiquaient plus des compétences, mais des moyens supplémentaires pour réaliser le nombre accru de leurs projets culturels par l'enrichissement de leur mission.

En deuxième lieu, la proposition du ministère de la Culture pour une nouvelle politique culturelle en 2012 réitéra la discussion sur la façon dont l'État planifiait l'attribution des compétences aux collectivités locales. Dans ce contexte, le ministère avait de son côté conscience de l'importance de garantir l'égalité des partenaires pour la mise en œuvre de cette coopération dans le respect à la fois de l'autonomie des régions et des communes (en termes de planification et de mise en place de la politique culturelle régionale) et celui des objectifs généraux du ministère au niveau national. À cet égard, l'État proposa «une forme de hiérarchie de fonction et

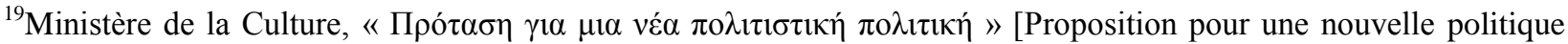
culturelle], Athènes, mars 2012, p. 62.
} 
non de tutelle» pour reprendre une formule de René Rizzardo (Rizzardo, 2005, p. 29). Cependant, le pilotage de l'action culturelle régionale tel que décrit dans l'encadré $\mathrm{n}^{\circ} 3$ connut un échec qui témoigna de la difficulté de l'État et des collectivités territoriales à passer à l'étape suivante de leur coopération, qui pourrait être caractérisée de phase de l'après-période contractuelle. Ainsi, le stade d'expérimentation, traduit par l'initiative de l'État en 2012 pour redéfinir le partenariat public, et par conséquent les objectifs d'une nouvelle démarche de décentralisation, ne dépassa pas la phase de la conception.

De leur côté, les fonctionnaires des structures culturelles régionales se montrèrent sceptiques quant à cette option du ministère. Leur argument reposait sur l'engendrement de nouvelles dépenses et la complexité de la mise en œuvre d'une politique culturelle régionale planifiée au niveau central. Plus précisément, ils soutenaient que dans ce cas, l'État serait dans l'obligation de créer des services pertinents, régionaux aussi bien que centraux, afin de servir une telle option politique (Kabouris, 2014). Ces services occasionneraient alors à l'État une surcharge financière importante, puisqu'ils créeraient un lourd mécanisme administratif dont l'efficacité était incertaine.

La crise politique et économique identifiée en Grèce dès la fin des années 2000 conduisit à une confusion dans la définition des priorités des politiques publiques. Dans ce contexte, nous n'avons pas connaissance de dépôt de propositions susceptibles d'inspirer la nouvelle étape de décentralisation culturelle. La limitation de la pratique de contrats de programme n'a pas contribué à la simplification des procédures partenariales dans le domaine culturel ou à l'invention de nouvelles. En outre, l'enrichissement des compétences des collectivités locales ne suscita pas l'amélioration des services culturels. Ainsi, bien que la nature de la mission des partenaires publics ait changé au niveau des responsabilités, leur coopération n'évolua pas en faveur du renforcement de la politique culturelle régionale.

\section{CONCLUSION}

La formule contractuelle, innovation politique et administrative, a conduit dans une certaine mesure à une unification des politiques culturelles menées par les villes. Cependant, la multiplication des contrats de programme depuis le milieu des années 1990 - à travers l'augmentation du nombre des Entreprises municipales de développement culturel, des DIPETHE et des structures d'EPDP - en conjonction avec la faible croissance du budget culturel à l'échelon central, a fragilisé la position de l'État vis-à-vis des villes. Ce sont ces 
conditions qui ont entraîné le retard de paiement des subventions étatiques et ont remis en cause la viabilité des institutions culturelles. L'abolition de l'EPDP en 2002 et la fusion des Entreprises municipales de développement culturel après 2006 ont affecté la crédibilité du pouvoir central. Le cas des DIPETHE excepté, l'État n'est intervenu que trop faiblement dans le budget de fonctionnement des structures culturelles de la périphérie.

En portant notre attention sur la politique de fusions et sur la réduction du nombre de structures culturelles locales, nous relevons une tendance à la marginalisation de la pratique du partenariat contractuel et par conséquent à la limitation de l'intervention de l'État à l'échelle locale. Dans cet esprit, la façon de coordonner l'action culturelle se différencie puisque le cadre contractuel, qui permettait au ministère de la Culture de mettre en œuvre ses propres politiques sectorielles, n'existe plus. Il est à noter toutefois que l'affaiblissement du rôle de l'État en tant qu'interlocuteur des collectivités locales trouve davantage sa source dans la stagnation de ses budgets que dans son évaluation des politiques et pratiques au cours des années précédentes. Ce constat nous conduit à réfléchir sur l'efficacité de nouveaux types d'intervention étatique dans l'exercice des politiques culturelles. Hormis la marque d'une attitude prudente de la part de l'État à l'égard de la gestion des structures culturelles par les collectivités locales, la logique de fusions n'a pas proposé un mode de fonctionnement alternatif susceptible de renforcer leur action. De plus, cette évolution n'a pas encouragé de manière sensible la cohérence d'interventions des différents échelons des collectivités territoriales (communes, régions).

Il s'agit donc d'une stratégie qui met l'accent sur une dimension fonctionnelle plutôt que politique de l'action des structures culturelles locales, et qui alimente un discours sur la marginalisation de la décentralisation en tant que priorité du pouvoir central. À cet égard, nous ne notons pas de redéfinition du rôle des protagonistes de l'action culturelle ou de leur mode d'action au niveau local mais du moins une difficulté de leur part à reconnaître les raisons qui empêchent l'évolution efficace de leurs politiques et pratiques.

\section{RÉFÉRENCES/REFERENCES}

Allies P. (1995), «Les régions du Sud et les programmes méditerranéens de l'Union européenne », Pôle Sud, n³, p. 141-146.

Autissier A-M. (2005), L'Europe de la culture, histoire(s) et enjeux, Paris, Maison des cultures du monde. 


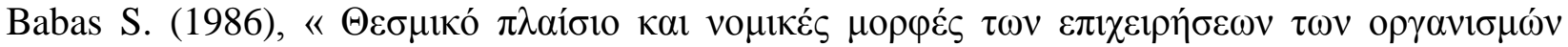

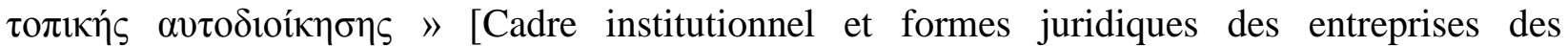

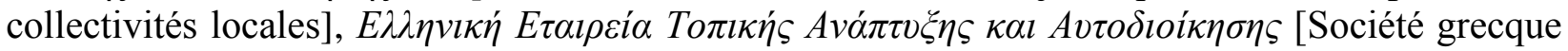
pour le développement local et le gouvernement local].

Contogeorgis G. (1995), « La politique locale entre la tendance centralisatrice de l'État grec et la dynamique communautaire : administration ou autonomie locale (et régionale) ? », Pôle Sud, $\mathrm{n}^{\circ}$ 3, p. 88-102.

Contogeorgis G. (2013), « Le problème grec », Pôle Sud, vol. 1, n 38, p. 79-86.

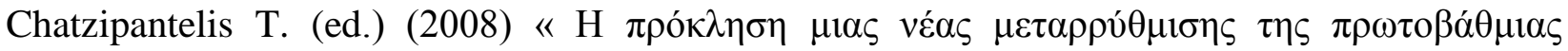

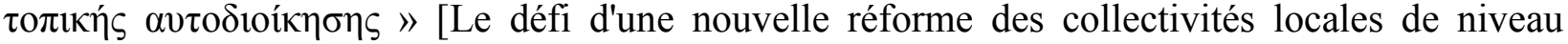

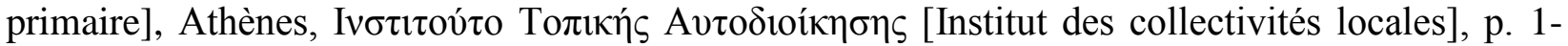
122, cf.http:www.kedke.gr/uploads2009/melethITA_proklisimetarrythmisis.doc

D’Angelo M. (2000), Politiques culturelles en Europe, la problématique locale, Strasbourg, Éd. du Conseil de l'Europe.

Dubois V., Bastien C., Freyermuth A., Matz K. (2012), Le politique, l'artiste et le gestionnaire, (re)configurations locales et (dé)politisation de la culture, Bellecombe-en-Bauges, Éd. du Croquant.

Dubois V., Négrier E. (1999), «L'institutionnalisation des politiques culturelles en Europe du Sud : éléments pour une approche comparée », Pôle Sud, n 10, p. 5-9.

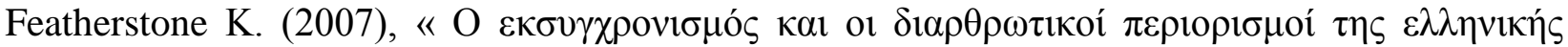
$\pi \mathrm{o} \imath \imath \imath \kappa \eta ́ \varsigma »[\mathrm{La}$ modernisation et les contraintes structurelles de la politique grecque], in

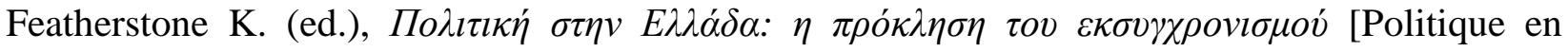
Grèce: le défi de la modernisation, Athènes, Okto.

Gaudin J-P. (2004), « XVII. La contractualisation des rapports entre l'État et les collectivités territoriales ", Annuaire des collectivités locales, tome 24. Réforme de la décentralisation, réforme de l'État. Régions et villes en Europe, p. 215-234.

Hassenteufel P., Surel Y. (2000), « Des politiques publiques comme les autres ? Construction de l'objet et outils d'analyse des politiques européennes », Politique européenne, vol. 1, $\mathrm{n}^{\circ} 1$, p. 8 24.

Hélie T. (2004), « Cultiver l'Europe. Éléments pour une approche localisée de l'européanisation des politiques culturelles », Politique européenne, vol. 12, n 1, p. 66-83.

Hlepas N-K. (2003), « Carte administrative et pouvoir politique : les enjeux de la décentralisation en Grèce », Pôle Sud, n 18. La Grèce du politique. p. 63-78. 


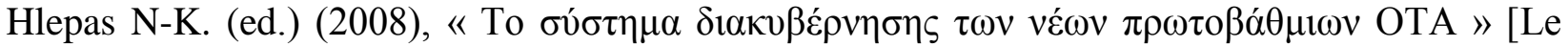
système de gouvernance des nouveaux Organismes d'Administration Locale (OTA) de niveau

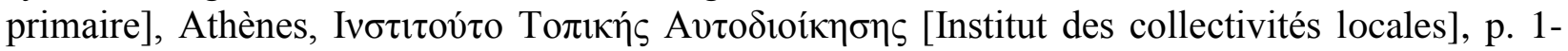
82,cf.http:www.kedke.gr/uploads2009/melethITA_diakyvernhshnevnOTA.doc

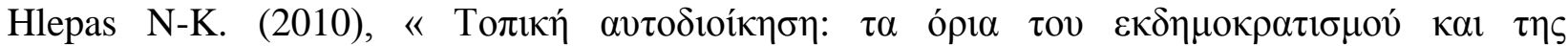

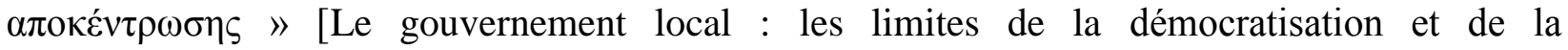

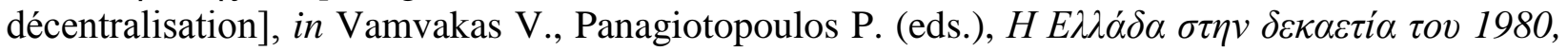

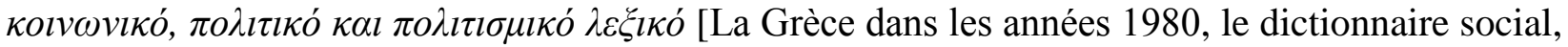
politique et culturel], Athènes, To perasma, p. 584-586.

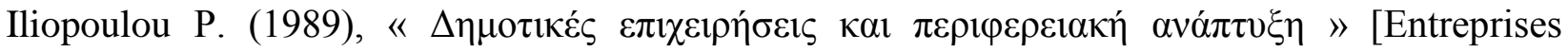

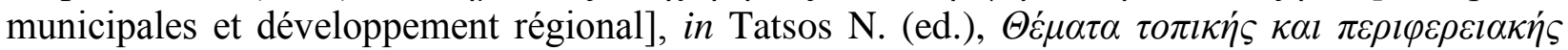
$\alpha v \alpha ́ \pi \tau v \xi \eta \varsigma$ [Questions de développement local et régional], Athènes, EETAA, p. 143-158.

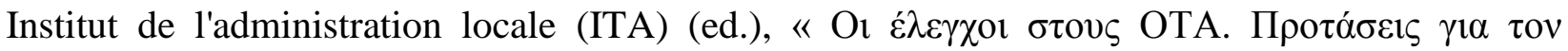

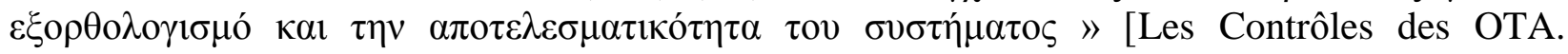
Propositions de rationalisation et d'efficacité du système], Athènes, le 22.06.2006.

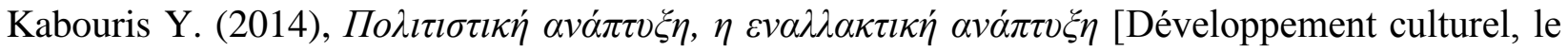
développement alternatif], Véria.

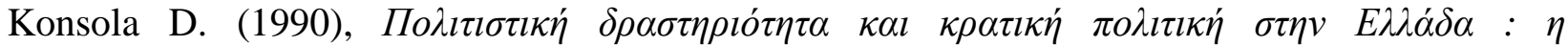
$\pi \varepsilon \rho \iota \varphi \varepsilon \rho \varepsilon \imath \alpha \kappa \eta \dot{~} \delta l \alpha ́ \sigma \tau \alpha \sigma \eta$ [Activité culturelle et politique étatique en Grèce : la dimension régionale], Athènes, Papazisis.

Konsola D. (1999), « La politique culturelle de la Grèce », Pôle Sud, vol. 10, n 1, p. 27-44.

Laborier P. (2003), " Historicité et sociologie de l'action publique » in Laborier P., Trom D. (dir.), Historicités de l'action publique, Paris, PUF-CURAPP, p. 419-462.

Le Gales P. (2002), Le retour des villes européennes. Sociétés urbaines, mondialisation, gouvernement et gouvernance, Paris, Presses de sciences po.

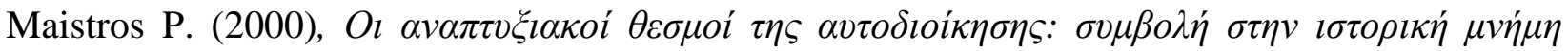
[Les institutions de développement de l'administration locale : une contribution à la mémoire historique], Athènes, Livanis-Nea Synora.

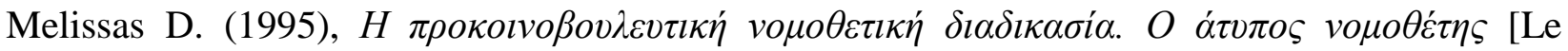
processus législatif pré-parlementaire. Le législateur atypique], Athènes, A.N. Sakkoulas.

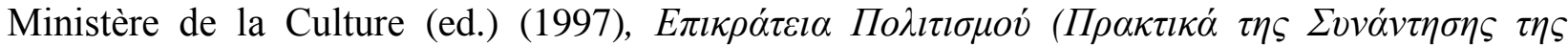

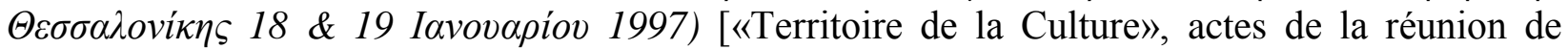
Thessalonique 18 \& 19 janvier 1997], Athènes.

Ministère de l'Intérieur de la Décentralisation et du Gouvernement Électronique (ed.) (2010), Rapport d'évaluation du projet de loi sur le Programme Kallikratis, Athènes. 
Négrier E. (2003), « Politiques culturelles territoriales : dernier inventaire avant décentralisation? ", Annuaire des collectivités locales, tome 23, p. 47-70.

Négrier E., Teillet P. (2014), « Le tournant instrumental des politiques culturelles », Pôle Sud, vol. $2, \mathrm{n}^{\circ} 41$, p. $83-100$.

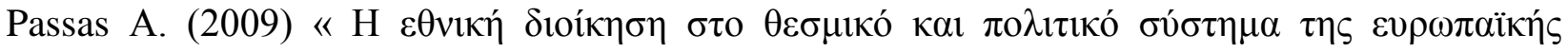

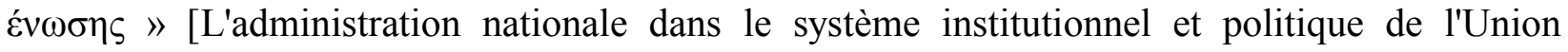

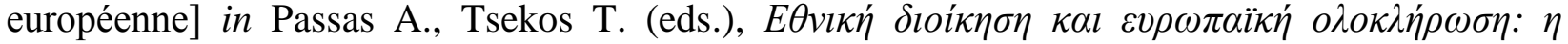
$\varepsilon \lambda \lambda \eta v \imath \kappa \eta \dot{~} \varepsilon \mu \pi \varepsilon \imath \rho i \alpha$ [Administration nationale et intégration européenne: l'expérience grecque], Athènes, éditions Papazisis.

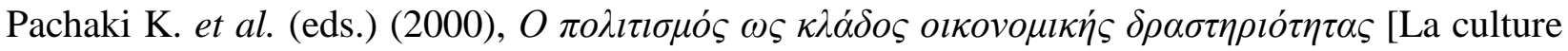

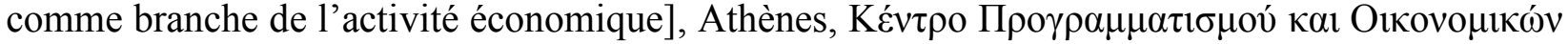

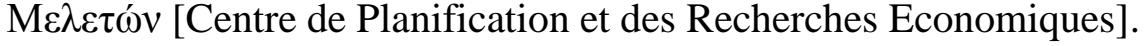

Peters G. (1998) « Managing Horizontal Government: The Politics of Co-Ordination », Canadian Public Administration, vol. 76, $\mathrm{n}^{\circ}$ 2, p. 295-311.

Pongy M. (1997), « Entre modèles nationaux et eurogroupes d'intérêts professionnels : l'action de l'Union dans la culture », Cultures \& Conflits, $\mathrm{n}^{\circ} 28$, p. 1-13.

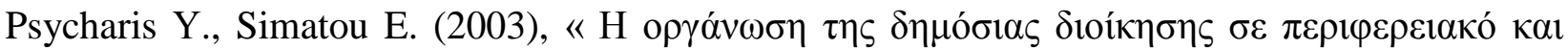

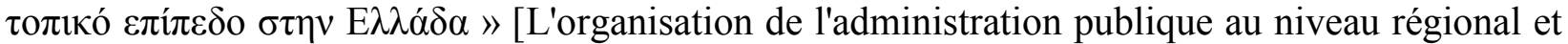

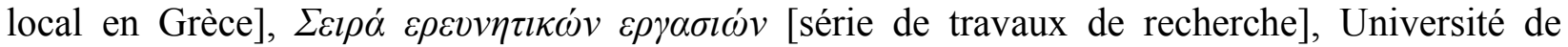
Thessalie, vol. 9(29).

Rizzardo R. (2005), « Décentraliser, c'est responsabiliser, mieux repartir les rôles, mieux coopérer », L'Observatoire, $\mathrm{n}^{\circ} 27$, p. 28-31.

Saez G. (2001), "Communes », in De Waresquiel E. (dir.), Dictionnaire des politiques culturelles de la France de 1959 à nos jours, Paris, CNRS Édition et Larousse-Bordas, p. 144148.

Saez G. (2005), « La gouvernance culturelle des villes dans l'espace politique européen », L'Observatoire, $\mathrm{n}^{\circ} 28$, p. 14-17.

Saurugger S., Surel Y. (2006), « L'européanisation comme processus de transfert de politique publique », Revue internationale de politique comparée, vol. 13, n 2, p. 179-211.

Schmidt V.A., Radaelli C.M. (2004), « Policy Change and Discourse in Europe: Conceptual and Methodological Issues », West European Politics, vol. 27, n² 2, p. 183-210.

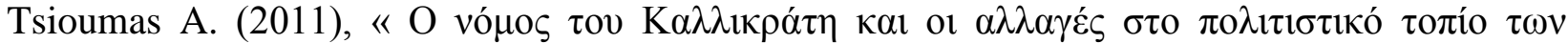
$\Delta \eta \dot{\mu} \omega \nu »[\mathrm{La}$ loi Kallikratis et les changements dans le paysage culturel des municipalités], directeur de mémoire, Ioannidis I., Athènes, École Nationale de l'Administration Locale.

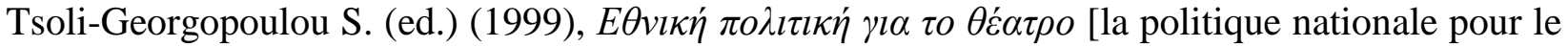
théâtre], Athènes, ministère de la Culture-Direction des Beaux-Arts. 


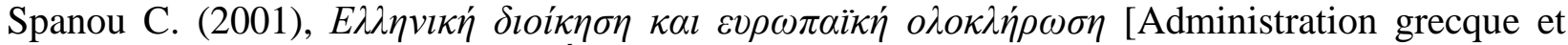
intégration européenne], Athènes, Éd. Papazisi.

Zorba M. (2011), « La politique culturelle de la Grèce. La culture comme objet de politique publique », in Poirrier P. (dir.), Pour une histoire des politiques culturelles dans le monde, 19452011, postface de Pierre-Michel Menger, Paris, Comité d'histoire du ministère de la Culture (Travaux et documents $n^{\circ} 28$ ), La Documentation française, p. 265-293.

\section{ANNEXES}

Tableau 2. Le réseau des Théâtres Municipaux Régionaux (1983-2010)

\begin{tabular}{|c|c|c|}
\hline THÉÂTRE & RÉGION & $\begin{array}{c}\text { DURÉE DE } \\
\text { FONCTIONNEMENT }\end{array}$ \\
\hline $\begin{array}{c}\text { Théâtre Municipal Régional de } \\
\text { Komotiní }\end{array}$ & Macédoine-Orientale-et-Thrace & 1984- \\
\hline $\begin{array}{l}\text { Théâtre Municipal Régional de } \\
\text { Kavala }\end{array}$ & Macédoine-Orientale-et-Thrace & 1992- \\
\hline Théâtre Municipal Régional de Véria & Macédoine-Centrale & 1983- \\
\hline $\begin{array}{c}\text { Théâtre Municipal Régional de } \\
\text { Serres }\end{array}$ & Macédoine-Centrale & 1984- \\
\hline $\begin{array}{l}\text { Théâtre Municipal Régional de } \\
\text { Kozani }\end{array}$ & Macédoine-Occidentale & 1997- \\
\hline $\begin{array}{c}\text { Théâtre Municipal Régional de } \\
\text { Ioannina }\end{array}$ & Épire & 1983- \\
\hline $\begin{array}{l}\text { Théâtre Municipal Régional de } \\
\text { Larissa }\end{array}$ & Thessalie & 1983- \\
\hline $\begin{array}{l}\text { Théâtre Municipal Régional de } \\
\text { Volos }\end{array}$ & Thessalie & 1994- \\
\hline $\begin{array}{c}\text { Théâtre Municipal Régional de } \\
\text { Corfou }\end{array}$ & Îles Ioniennes & $1997-$ \\
\hline $\begin{array}{c}\text { Théâtre Municipal Régional de } \\
\text { Agrínion }\end{array}$ & Grèce Occidentale & 1983- \\
\hline $\begin{array}{l}\text { Théâtre Municipal Régional de } \\
\text { Patras }\end{array}$ & Grèce Occidentale & 1987- \\
\hline $\begin{array}{c}\text { Théâtre Municipal Régional de } \\
\text { Roumélie (Lamía) }\end{array}$ & Grèce-Centrale & 1985- \\
\hline $\begin{array}{l}\text { Théâtre Municipal Régional de } \\
\text { Kalamata }\end{array}$ & Péloponnèse & 1983- \\
\hline $\begin{array}{l}\text { Théâtre Municipal Régional d'Égée- } \\
\text { Septentrionale (Chios) }\end{array}$ & Égée-Septentrionale & 1995- \\
\hline $\begin{array}{c}\text { Théâtre Municipal Régional de } \\
\text { Rhodes }\end{array}$ & Égée-Méridionale & $1987-2010$ \\
\hline $\begin{array}{l}\text { Théâtre Municipal Régional de Crète } \\
\text { (La Canée) }\end{array}$ & Crète & 1983- \\
\hline
\end{tabular}

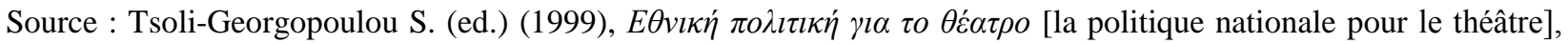
Athènes, ministère de la Culture-Direction des Beaux-Arts. p. 56-57, [traitement des données par l'auteure de l'article]. 
Tableau 3. Les structures culturelles du Réseau Culturel National des Villes (EPDP)

\begin{tabular}{|c|c|}
\hline Région & Structure culturelle \\
\hline Macédoine Orientale et Thrace & $\begin{array}{c}\text { Centre Audiovisuel (Alexandroupolis) } \\
\text {-Centre de Rencontres Internationales sur les Arts } \\
\text { Plastiques (Didymoteicho) } \\
\text { •Festival des Films de Courts Métrages (Drama) } \\
\text { •Centre des Arts Folkloriques (Komotini) }\end{array}$ \\
\hline Macédoine Centrale & $\begin{array}{c}\text {-Centre d'Initiatives Culturelles et de la Communication } \\
\text { (Veria) }\end{array}$ \\
\hline Macédoine Occidentale & $\begin{array}{c}\text { •Centre d'Art Byzantin (Kastoria) } \\
\text { •Institut du Livre et de la Lecture (Kozani) } \\
\text { •Institut Ethnographique de l'Europe du Sud (Florina) }\end{array}$ \\
\hline Épire & $\begin{array}{c}\text {-Centre de Recherche de la Musique d'Épire et des } \\
\text { Balkans (Ioannina) }\end{array}$ \\
\hline Thessalie & $\begin{array}{l}\text {-Centre de Théâtre Musical (Volos) } \\
\text {-Centre de Photographie (Skopelos) } \\
\text { •Centre d'Art Contemporain (Larissa) }\end{array}$ \\
\hline îles Ioniennes & -Centre d'Activité Chorale (Argostoli-Lixouri) \\
\hline Grèce Occidentale & -Chemins de chanson (Patras) \\
\hline Grèce Centrale & - Centre de Musique Méditerranéen (Lamia) \\
\hline Péloponnèse & $\begin{array}{l}\text {-Centre International de Danse (Kalamata) } \\
\text {-Centre d'Activités Artistiques (Tripoli) }\end{array}$ \\
\hline Crète & $\begin{array}{l}\text { •Centre d'Art Contemporain (Rethymno) } \\
\text { •Centre d'Architecture Méditerranéenne (Le Canée) }\end{array}$ \\
\hline
\end{tabular}

Source: $\triangle \rho \alpha \dot{\sigma \varepsilon \varepsilon l \varsigma ~ \pi о \lambda \imath \tau \imath \sigma \mu o v ́ ~ \sigma \tau \eta v ~ \pi \varepsilon \rho \imath \varphi \varepsilon ́ \rho \varepsilon \imath \alpha ~[A c t i o n s ~ c u l t u r e l l e s ~ e n ~ r e ́ g i o n] ~(1997), ~ C e n t r e ~ d ' I n i t i a t i v e s ~ C u l t u r e l l e s ~ e t ~}$ de la Communication, $1^{\text {er }}$ édition, Vèria, [traitement des données par l'auteure de l'article]. Ce tableau illustre les structures créées durant la première phase d'institution (1994-1996) pendant le ministère de Thanos Miktroutsikos.

\section{Encadré 4. Les domaines d'action de l'Organisme Artistique de la Municipalité de Volos (1988-2008)}

\section{-Secteur de la musique}

$\downarrow$

- Conservatoire municipal (1988)

- Orchestre symphonique (1992)

- Orchestre symphonique des jeunes (1998)

-Centres de l'enfance

et de la jeunesse (1985)
- Secteur de la danse

- École de danse (1990)

-Secteur audiovisuel

$\downarrow$

- Station de radio (1998)
-Secteur des arts plastiques

$\downarrow$

- Expositions de peinture

-Atelier d'arts plastiques (1988)

Source : archives du ministère de la Culture, Direction Générale de la Culture Contemporaine, Département des actions culturelles, Dossier thématique - Volos/ Département du Théâtre et de la Danse, Dossier thématique DIPETHE Volos. 